\title{
Design of Magnetic Actuator Intended for Artificial Heart Drive
}

\author{
H. Saotome, K. Shimizu, and T. Okada \\ Faculty of Engineering, Chiba University, 1-33 Yayoi, Inage, Chiba 263-8522
}

With the goal of developing an artificial heart, a linear motion magnetic actuator has been produced and applied to a pump. The actuator is composed of two ring-shaped Nd-Fe-B magnets that face each other, the outer and immer diameters and thickness of which are $63 \mathrm{~mm}, 17 \mathrm{~mm}$ and $3 \mathrm{~mm}$, respectively. Rotating one of the magnets circumferentially via a motor produces reciprocating forces on the other magnet. The pumping motion is performed using the power of the reciprocating magnet. Because the actuator uses no windings, no copper loss, which causes heat generation in the body, is generated. The output powers of the actuator and the pump reached $1.03 \mathrm{~W}$ and $0.65 \mathrm{~W}$, respectively.

Key words: actuator, linear motion, artificial heart, $\mathrm{Nd}-\mathrm{Fe}-\mathrm{B}$ magnet, pump

\section{Introduction}

Strong metal magnets with a large energy product enable the development of various types of magnetic actuators $^{1), 2)}$. Some of the magnetic actuators have simple structures with no windings. In these cases, the magnetic actuators are driven by the magnetic fields generated by permanent magnets, and/or coil currents at a distance. In addition, compared to the conventional type magnetic actuators with iron yokes and solenoids, actuators with metal magnets offer the advantage of push and pull forces whereas only pull forces occur magnetically on the iron yokes.

Utilizing the push and pull forces occurring on metal magnets, the authors have developed pumps that are driven by linear motion actuators composed of two ring-shaped $\mathrm{Nd}-\mathrm{Fe}-\mathrm{B}$ magnets that face each other ${ }^{3), 4)}$. The actuator has no windings and yields no copper loss, and therefore is suitable for driving an artificial heart because of its low heat generation in the body. This paper presents experimentally obtained output characteristics of a newly developed actuator and pump.

\section{Pump structure}

Using two ring-shaped Nd-Fe-B magnets like those shown in Fig. 1, a pump was constructed like that shown in Fig. 2. The $\mathrm{N}$ and $\mathrm{S}$ poles of the two magnets occupy the surface equally. The reverse side of the N-pole area is the S-pole, and vice versa. The two magnets are set on the same axis and face each other. Iron yokes are fixed to the outsides of the magnets in order to strengthen the magnetic field between the magnets. By rotating the left-hand magnet in Fig. 1 circumferentially, push and pull forces are exerted on the right-hand magnet. When the right-hand

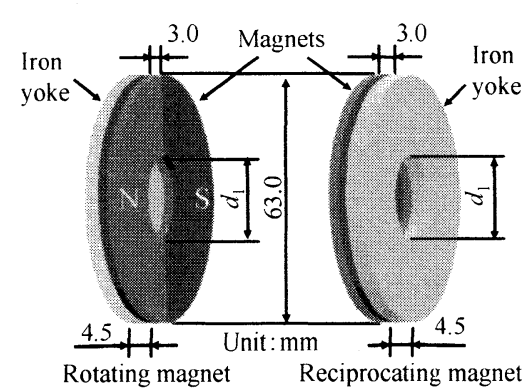

Fig.1 Two ring-shaped Nd-Fe-B magnets face each other.

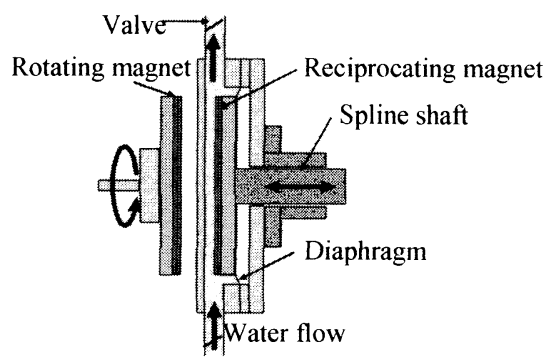

Fig.2 Pump structure.

magnet is restricted to linear motion along the axis by a spline shaft, as shown in Fig. 2, the magnet moves left and right alternately. As a result, the mechanical power driving the rotating magnet is transferred to the linear motion power.

Because the magnetic field penetrates the skin of a human body, the rotating magnet driven by a motor can be located outside the body when the pump is applied to an artificial heart. In this system, no windings, which cause copper loss, are required to be buried in the body: only the mechanical system containing the right-hand magnet need be implanted. Therefore, it is expected that an artificial heart with low heat generation in the body can be realized.

The magnetic force of the magnets depends on their size. In the present paper, the outer diameter and thickness of the magnets are fixed to $63 \mathrm{~mm}$ and $3 \mathrm{~mm}$, respectively, which are the same dimensions as the magnets used in a previous pump ${ }^{4}$. In the actuator used in the previous pump, the inner diameter of the magnets was $39 \mathrm{~mm}$. Increased output power from the actuator is expected by changing the inner diameter. The inner diameter of the magnets applying to a new actuator, $d_{1}$, is designed by the following steps that have been devised.

Assuming that the thickness of the skin is approximately 6-7 $\mathrm{mm}$, the minimum space between the magnets, $x_{\min }$, shown in Fig. 3, should be maintained to be at least $10 \mathrm{~mm}$. The maximum output pressure of the pump must be less 


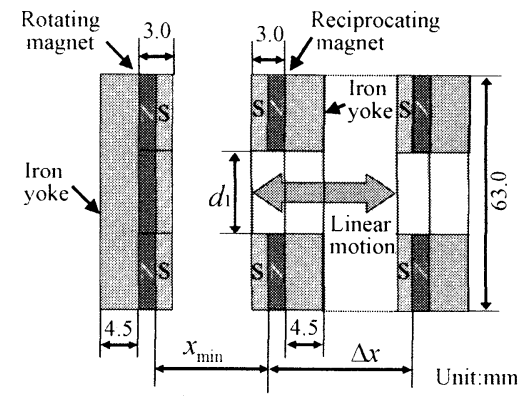

Fig.3 Stroke of the linear motion magnet, $\Delta x$.

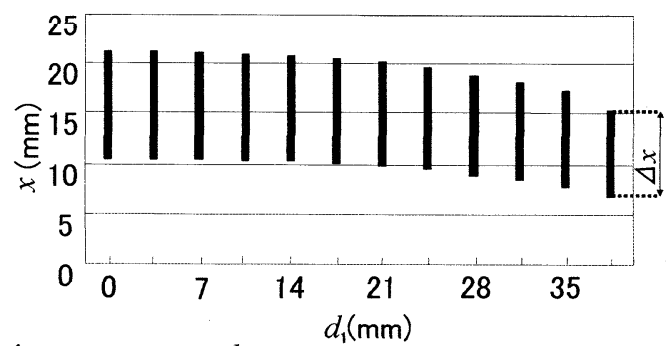

Fig.4 $\Delta x$ versus $d_{1}$.

than $150 \mathrm{mmHg}(20 \mathrm{kPa})$ because of the allowed blood pressure, i.e., the force on the magnets must be less than $220 \mathrm{~N}$ for the size shown in Fig. 1. On the other hand, here we set the minimum repulsive and attractive forces as 15 $\mathrm{N}$ and $55 \mathrm{~N}$, respectively, for the size, although they provide less power than a real heart. In other words, at the right-hand side end of the stroke of the reciprocating magnet shown in Figs. 2 and 3, the forces toward the right and left directions are maintained to be at least $15 \mathrm{~N}$ and $55 \mathrm{~N}$, respectively, for continuous motion under load.

By three-dimensional static magnetic field computations using an FEM software package, JMAG ${ }^{5}$, the magnetic force on the reciprocating magnet has been evaluated as a function of $d_{1}$. By the force value limitations mentioned above, strokes of the reciprocating magnet have been chosen as shown in Fig. 4 where $x=x_{\min }+\Delta x$ and $\Delta x$ is the stroke. Based on the condition of $x_{\min } \geq 10 \mathrm{~mm}$, we have set $d_{1}=17 \mathrm{~mm}$. As a result, $\Delta x=10 \mathrm{~mm}$. We make a hole in the reciprocating magnet simply to reduce its weight. In the range of $d_{1}<17 \mathrm{~mm}$, the magnetic force increase resulting from a decrease of $d_{1}$ is too small to extend the available stroke length $\Delta x$ considerably, as shown in Fig. 4. The thickness of the iron yokes, fixed to the magnets, is estimated as $4.5 \mathrm{~mm}$ using JMAG and avoiding their magnetic saturation.

\section{Output characteristics}

The magnetic force $F$ on the reciprocating magnet has been measured with variations of $x$ and the angle of the rotating magnet, $\theta$, as shown in Fig. 5, where $\theta=0^{\circ}$ is defined as the angle at which the repulsive force is maximum, i.e., the same magnetic poles are completely facing each other. The positive and negative signs of the force in Fig. 5 indicate repulsive and attractive, respectively. The fact that the force is biased toward

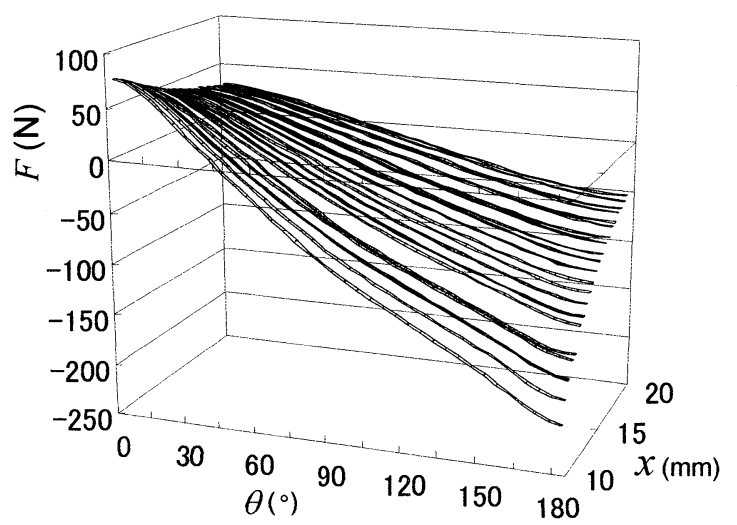

Fig.5 Measured force between the magnets.

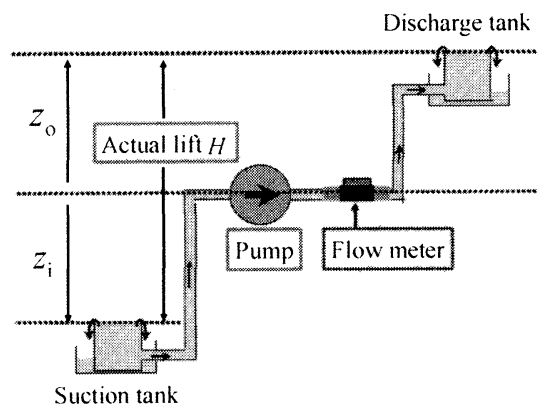

Fig.6 Load test for the pump.

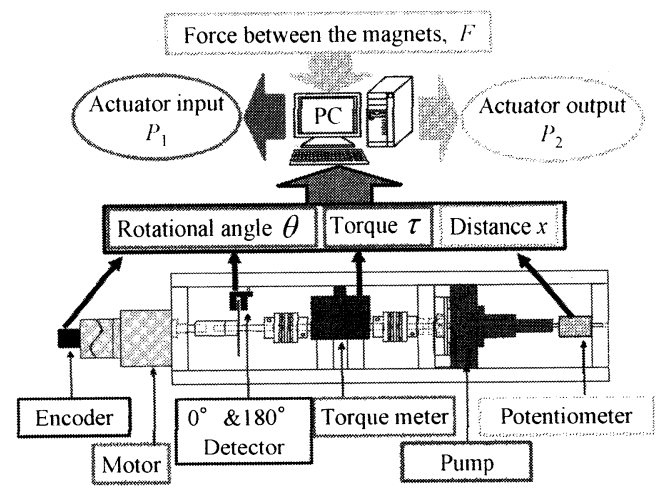

Fig.7 Measurement system of the input and output powers of the actuator.

negative is caused by the attractive forces on the iron yokes.

The pump has been loaded with tap water, as shown in Fig. 6. During the pumping motion, the torque $\tau$ on the rotating magnet and the distance $x$ between the magnets are measured by the measurement system shown in Fig. 7 . On the load tests, the rotating magnet driven by a motor does not revolve at a constant speed, but stops at $\theta=0^{\circ}$ and $\theta=180^{\circ}$ then resumes revolving when the reciprocating magnet reaches the ends of the strokes. This driving method increases the output power of the pump compared to a constant revolution drive ${ }^{4)}$. by

The driving power of the rotating magnet, $P_{1}$, is given 


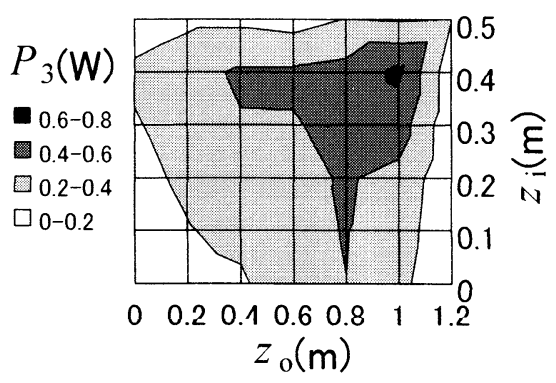

Fig.8 $P_{3}$ versus water pressure.

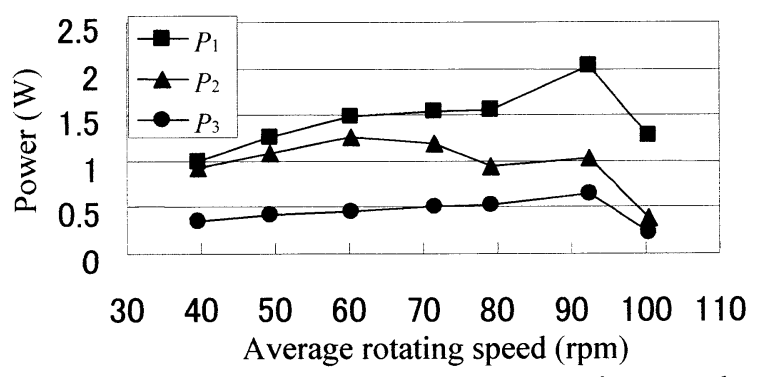

Fig.9 $\quad P_{1}, P_{2}$ and $P_{3}$ versus the average rotating speed.

$$
P_{1}=\frac{1}{T} \int_{0}^{2 \pi} \tau(\theta) d \theta
$$

where $T$ denotes the period of the revolution. The output power of the reciprocating magnet, $P_{2}$, is given by

$$
P_{2}=\frac{1}{T} \int F(x) d x,
$$

where $F$ is obtained from the data shown in Fig. 5 with the measured angle $\theta$. Using the measured instantaneous water flow $q\left(\mathrm{~m}^{3} / \mathrm{s}\right)$, the output power of the pump, $P_{3}$, is given by

$$
P_{3}=\rho g H \frac{1}{T} \int_{0}^{T} q(t) d t,
$$

where $\rho$ is the density of water, $g$ is the gravity and $H$ $=z_{\mathrm{i}}+z_{\mathrm{o}}$.

The output characteristics of the pump have been obtained, as shown in Fig. 8, where the average rotating speed per revolution is $92 \mathrm{rpm}$. The maximum output power of $0.65 \mathrm{~W}$, which is more than twice as large as that of the previous pump, has been obtained. The dependence of $P_{1}, P_{2}$ and $P_{3}$ on the average rotating speed is shown in Fig. 9 where $z_{\mathrm{i}}=0.4 \mathrm{~m}$ and $z_{\mathrm{o}}=1.0 \mathrm{~m}$, respectively. The actuator output $P_{2}$ is $1.03 \mathrm{~W}$ when $P_{3}$ is $0.65 \mathrm{~W}$. The maximum value of $P_{2}, 1.27 \mathrm{~W}$, is obtained at $60 \mathrm{rpm}$. The pump output $P_{3}$ is proportional to the rotating speed, but declines at $100 \mathrm{rpm}$ because the reciprocating magnet turns before reaching the ends of the strokes.

The actuator loss $P_{1}-P_{2}$ is primarily caused by the iron loss in the yokes ${ }^{4}$. For example, $P_{1}-P_{2}=0.22 \mathrm{~W}$ at $60 \mathrm{rpm}$. Using a low-iron-loss material for the yokes, the actuator efficiency is expected to increase. The other loss in $P_{1}-P_{2}$ is the friction loss caused in the spline shaft which prevents the reciprocating magnet from rotating. However, the friction loss is assumed to be considerably smaller than the iron loss.

Supplying the pump output of $0.65 \mathrm{~W}$, the total loss $P_{1}$ $-P_{3}$ is $1.38 \mathrm{~W}$. The total efficiency $P_{1} / P_{3}$ is $32 \%$, which is double that of the conventionally proposed motor-driven total artificial heart, in which pump output versus motor input is approximately $20 \%$ and the power transfer ratio via a percutaneous transformer to the motor is approximately $80 \%$ due to the iron and copper losses as well as the power converter loss ${ }^{6}$.

\section{Conclusion}

We have discussed an actuator design with two ring-shaped magnets when it is applied to an artificial heart. In order to increase the output power of the actuator, the relationship between the stroke and the inner diameter of the reciprocating magnet has been evaluated with numerical magnetic field computations. The output characteristics of a pump that was developed using the newly designed actuator have been experimentally evaluated.

The required maximum output blood flow of the artificial heart is 8 liter per minute with the pressure $100 \sim$ $120 \mathrm{mmHg}$. The maximum output power corresponds to more than $2 \mathrm{~W}$. Its size is required to reduce under 0.9 liter $^{6}$. Although the obtained pump power of $0.65 \mathrm{~W}$ in this paper is only half the value of the actual heart output power needed when resting, we intend to continue to study the actuator because it offers important benefits of simple structure and no copper loss.

Acknowledgements This study was financially supported by a Grant-in-Aid for Fundamental Scientific Research from the Japan Society for the Promotion of Science, and by the Electro-Mechanical Technology Advancing Foundation.

\section{References}

1) M. Sendoh, K. Ishiyama, K. Arai, M. Jojo, F. Sato, and H. Matsuki: IEEE Trans. Magn. 38, 3359(2002).

2) H. Saotome, T. Okubo, and Y. Ikeda: IEEE Trans. Magn. 38, 3009(2002).

3) H. Saotome, T. Hagiwara, and Y. Sato: J. Appl. Phys., 83, 6420(1998).

4) H. Saotome, K. Imamura, Y. Sato, and O. Saito: T.IEE Japan, 120-A, 1038(2000).

5) http://www.jri.co.jp/pro-eng/jmag/e/jmg/index.html

6)Electromagnetic Artificial Heart, IEEJ ed., p.28, p.104, p.15, (Corona Publishing Co., Ltd., Tokyo, 1994) [in Japanese].

Received Dec. 3, 2003; Accepted Mar. 11, 2004. 